\title{
Checklist de Simuliidae (Insecta, Diptera) do Estado de São Paulo, Brasil
}

\author{
Mateus Pepinelli ${ }^{1,2}$ \\ ${ }^{1}$ Laboratório de Entomologia Aquática, Faculdade de Filosofia, Ciências e Letras de Ribeirão Preto - FFCLRP, \\ Universidade de São Paulo - USP, Av. Bandeirantes, 3900, CEP 14040-901, \\ Monte Alegre, Ribeirão Preto, SP, Brasil \\ ${ }^{2}$ Autor para correspondência: Mateus Pepinelli, e-mail: mateuspepi@yahoo.com.br
}

PEPINELLI, M. Checklist of Simuliidae (Insecta, Diptera) from São Paulo State, Brazil. Biota Neotrop. 11(1a): http://www.biotaneotropica.org.br/v11n1a/en/abstract?inventory+bn0341101a2011.

Abstract: In this paper it is provided a checklist of Simuliidae species in the State of São Paulo, Brazil. There are 52 species recorded for the state, which represents more than $50 \%$ of all black fly known species in Brazil. It is also presented data about the Simuliidae species richness in the State of São Paulo as well as in different regions of Brazil.

Keywords: Simuliidae, biodiversity of the State of São Paulo, BIOTA/FAPESP Program.

Number of species: In the world: 2,114 (12 being fossils), in Brazil: 92, estimated in São Paulo State: 52.

PEPINELLI, M. Checklist dos Simuliidae (Insecta, Diptera) do Estado de São Paulo, Brasil. Biota Neotrop. 11(1a): http://www.biotaneotropica.org.br/v11n1a/pt/abstract?inventory+bn0341101a2011.

Resumo: Nesse artigo é fornecida uma lista de espécies de Simuliidae do Estado de São Paulo. Até o momento 52 espécies estão registradas para o estado, o que representa mais de $50 \%$ de todas as espécies de borrachudos conhecidas para o Brasil. São discutidos aspectos da riqueza de espécies no Estado de São Paulo e em diferentes regiões brasileiras.

Palavras-chave: Simuliidae, biota paulista, Programa BIOTA/FAPESP.

Número de espécies: No mundo: 2.114 (sendo 12 fósseis), no Brasil: 92, estimadas no estado de São Paulo: 52. 


\section{Introdução}

Pertencentes à família Simuliidae, os borrachudos ocupam a trigésima posição em número de espécies dentre as 130 famílias de Diptera (Adler et al. 2004). De acordo com a última contagem, 2114 espécies válidas (incluindo 12 fósseis) estão descritas para todo o planeta (Adler \& Crosskey 2010), o que representa aproximadamente $2 \%$ do total de espécies de Diptera descritas. Os borrachudos estão presentes em ambientes lóticos do mundo todo, exceto na Antártica. Sua abundância, ampla distribuição geográfica, importância médica e econômica e, principalmente, devido ao fato de algumas espécies picarem os humanos, conferem a esse grupo de insetos um privilegiado reconhecimento perante o público (Adler et al. 2004).

Nas regiões Norte e Nordeste do Brasil esses insetos são conhecidos como pium. No resto do país são chamados de borrachudos. No meio acadêmico e técnico muitas vezes é utilizado o jargão "simulídeo". Esse grupo de insetos vive em dois ambientes completamente distintos: o meio aquático e o terrestre. No ambiente aquático são organismos extremamente importantes e benéficos, pois fazem parte da base da cadeia alimentar. Suas larvas vivem aderidas a um substrato (folhas, galhos, pedras) e são filtradoras alimentando-se de partículas orgânicas finas (FPOM) dissolvidas e em suspensão na água, como algas e detritos finos. No ambiente terrestre são reconhecidos como insetos pestes e incômodos. Os adultos são diurnos, ambos os sexos alimentam-se de néctar de plantas e flores e somente as fêmeas de algumas espécies são antropofílicas, isto é, alimentam-se de sangue de humanos (Coscarón \& Coscarón-Arias 2007). As fêmeas (geralmente após o repasto sanguiíneo) depositam os ovos em vários tipos de substratos submersos ou sobre a água.

Os borrachudos geralmente estão presentes em elevadas densidades em córregos, rios, saídas de represas, de lagos e de reservatórios, pois é onde as formas imaturas se criam. Em condições favoráveis as larvas podem ocorrer em grandes quantidades, com densidades populacionais acima de 1 milhão de indivíduos $/ \mathrm{m}^{2}$ (Currie \& Adler 2008). Sob tais circunstâncias são importantes fontes de alimento para outros invertebrados (ex: plecópteros) e vertebrados (ex: peixes). Além disso, as larvas de Simuliidae têm um papel fundamental no processamento de matéria orgânica de córregos e rios, pois durante o processo de alimentação (filtração) as larvas de borrachudos transformam as partículas finas (FPOM) dissolvidas e suspensas na água em pelotas fecais maiores. Tais pelotas afundam rapidamente servindo de comida para organismos coletadores-juntadores. A importância e a magnitude desse processo foram comprovadas por Malmqvist et al. (2001), cuja estimativa de transporte de pelotas fecais eliminadas por larvas de Simuliidae atingiu o valor máximo de 429 t (peso seco) em apenas 1 dia, durante a vazão máxima de um trecho do rio Vindel, na Suécia. Essa matéria orgânica reciclada fornece alimentos para invertebrados e microorganismos e, potencialmente, pode fertilizar vales de rios (Malmqvist et al. 2004).

\section{Metodologia}

Na elaboração dessa lista de espécies de Simuliidae do Estado de São Paulo, foram consultadas várias fontes, com destaque para: Adler \& Crosskey (2010), Coscarón \& Coscarón-Arias (2007) e Pepinelli(2008). Anteriormente ao Programa BIOTA/FAPESP, havia o registro de 40 espécies de Simuliidae para o Estado de São Paulo. Todas as novas informações publicadas acerca de novas espécies ou de novos registros de Simuliidae para o estado de São Paulo desde que o Programa BIOTA/FAPESP foi lançado em 1999 foram oriundas de dois projetos de pesquisa, uma dissertação de mestrado e uma tese de doutorado, ambas dentro de projetos temáticos financiados pelo Programa BIOTA/FAPESP.

Durante tais projetos, várias localidades de coleta foram visitadas (mais de 150 rios e córregos) abrangendo todas as 22 unidades de gerenciamento de recursos hídricos do Estado de São Paulo (UGRH), tanto em áreas florestadas quanto em áreas de agricultura, pastagens, entre outras.

Em todas as localidades (córregos, rios, nascentes) visitadas foram encontradas larvas de borrachudos, o que demonstra a ampla distribuição geográfica do grupo e a participação dos imaturos como membros regulares das comunidades de insetos aquáticos de sistemas lóticos. No total, foram coletadas 43 espécies de Simuliidae nos 151 córregos e rios amostrados durante este estudo. As demais nove espécies da lista (totalizando 52 (Tabela 1)) não foram recoletadas e algumas são conhecidas apenas de suas coletas originais.

A riqueza de espécies foi maior em córregos localizados na região da Mata Atlântica, onde foram coletadas e identificadas 38 espécies em 48 córregos florestados situados em Parques Estaduais e remanescentes de Florestas do Estado de São Paulo. Já nos córregos localizados fora das áreas florestadas (103 localidades) a riqueza de espécies diminui para 23. Destes 103 córregos, 43 não possuíam mata ripária e apresentavam problemas de assoreamento. Nesses 43 córregos a riqueza foi de apenas 16 espécies, ou seja, menos da metade das espécies coletadas em ambientes preservados.

O número de espécies em um único córrego variou de 1 a 9 (média $=2,7$ ). Considerando apenas córregos localizados em áreas florestadas, a riqueza média é um pouco mais elevada (3,2), enquanto o número médio nos demais córregos foi de 2,2. Entre os 43 córregos mais impactados o número médio foi de 1,7. Estudos similares realizados na Amazônia Central resultaram na coleta de 11 espécies nos 58 córregos estudados, com o número máximo de 4 espécies em um único córrego e média de 2,3 espécies por córrego (Hamada et al. 2002). Strieder (2002) coletou e identificou 14 espécies de Simuliidae em oito cursos d'água na Bacia do rio Maquiné, no Estado do Rio Grande do Sul. Na mesma região, Strieder et al. (2002) identificaram um número maior de espécies (24) em 48 localidades ao longo da bacia hidrográfica do Rio dos Sinos.

O grande número de espécies de borrachudos registrados para o Estado de São Paulo em comparação com outras regiões do Brasil, em parte é resultado de um maior esforço amostral e da participação de vários especialistas de Simuliidae que trabalharam em instituições de São Paulo ao longo das últimas décadas, principalmente estudando a diversidade desse grupo em córregos de Mata Atlântica. Além disso, a abrangência das coletas realizadas durante os últimos anos, com um elevado número de localidades visitadas em diferentes regiões e, sobretudo pela grande variedade de ambientes visitados, incluindo rios e córregos de diferentes ordens, nascentes localizadas em elevadas altitudes, etc, refletem os resultados obtidos até o momento.

Em relação aos demais estados do Brasil, a riqueza de espécies de Simuliidae é mais alta no Estado de São Paulo, seguido pelo Estado do Rio de Janeiro, Minas Gerais e Rio Grande do Sul (Figura 1). Já o Nordeste é a região mais desprovida de informações sobre Simuliidae, com detaque para o Estado da Bahia com o maior número de registro de espécies (20). Tais dados relevam a necessidade de maiores estudos para atualizar o estado do conhecimento da biodiversidade do nosso país, principalmente na região Nordeste. 
Tabela 1. Lista de espécies de Simuliidae registradas para o Estado de São Paulo, Brasil.

Table 1. Checklist of Simuliidae species recorded for the State of São Paulo, Brazil.

Lista das espécies de Simuliidae do Estado de São Paulo

Gênero LUTZSIMULIUM d'Andretta \& d'Andretta, 1947 [1946]

Lutzsimulium hirticosta (Lutz, 1909)

Lutzsimulium pernigrum (Lutz, 1910)

Gênero SIMULIUM Latreille, 1802

Subgênero ASPATHIA Enderlein, 1935

Simulium metallicum Bellardi, 1859 [1861] (complexo de espécies)

Subgênero CHIROSTILBIA Enderlein, 1821

Simulium bifenestratum Hamada \& Pepinelli, 2004

Simulium dekeyseri Shelley \& Py-Daniel, 1981

Simulium distinctum Lutz, 1910

Simulium empascae Py-Daniel \& Moreira, 1988

Simulium friedlanderi Py-Daniel, 1987

Simulium obesum Vulcano, 1959

Simulium pertinax Kollar, 1832

Simulium riograndense Py-Daniel, Souza \& Caldas, 1988

Simulium serranus Coscarón, 1981

Simulium spinibranchium Lutz, 1910

Simulium subpallidum Lutz, 1910

Subgênero HEMICNETHA Enderlein, 1934

Simulium brachycladum Lutz \& Pinto, 1932 [1931]

Simulium rubrithorax Lutz, 1909

Subgênero INAEQUALIUM Coscarón \& Wygodzinsky, 1984

Simulium botulibranchium Lutz, 1910

Simulium clavibranchium Lutz, 1910

Simulium diversibranchium Lutz, 1910

Simulium inaequale (Paterson \& Shannon, 1927)

Simulium mariavulcanoae Coscarón \& Wygodzinsky, 1984

Simulium marins Pepinelli, Hamada \& Currie, 2009

Simulium rappae Py-Daniel \& Coscarón, 1982

Simulium subnigrum Lutz, 1910

Simulium travassosi d'Andretta \& d'Andretta, 1947

Subgênero NOTOLEPRIA Enderlein, 1930

Simulium exiguum Roubaud, 1906 (complexo de espécies)

Simulium incertum Lutz, 1910

Simulium paraguayense Schrottky, 1909

Subgênero PSARONIOCOMPSA Enderlein, 1934

Simulium anamariae Vulcano, 1962

Simulium angrense Pinto, 1932 [1931]

Simulium auristriatum Lutz, 1910

Simulium brevifurcatum Lutz, 1910

Simulium incrustatum Lutz, 1910 (complexo de espécies)

Simulium jujuyense Paterson \& Shannon, 1927

Simulium limbatum Knab, 1915

Simulium lutzi Knab, 1913

Simulium minusculum Lutz, 1910

Simulium oyapockense Floch \& Abonnenc, 1946 (complexo de espécies)

Simulium stellatum Gil-Azevedo, Figueiró \& Maia-Herzog, 2005

Simulium varians Lutz, 1909

Subgênero ECTEMNASPIS Enderlein, 1934

Simulium dinellii (Joan, 1912)

Simulium lutzianum Pinto, 1932 [1931] (complexo de espécies) 
Lista das espécies de Simuliidae do Estado de São Paulo

Simulium ochraceum Walker, 1861 (complexo de espécies)

Simulium perflavum Roubaud, 1906

Simulium shewellianum Coscarón, 1985

Subgênero TRICHODAGMIA Enderlein, 1934

Simulium nigrimanum Macquart, 1838

Subgênero THYRSOPELMA Enderlein, 1934

Simulium duodenicornium Pepinelli, Hamada \& Trivinho-Strixino, 2005

Simulium guianense Wise, 1911 [1912] (complexo de espécies)

Simulium hirtipupa Lutz, 1910

Simulium itaunense D’Andretta \& González, 1964

Simulium orbitale Lutz, 1910

Simulium scutistriatum Lutz, 1909

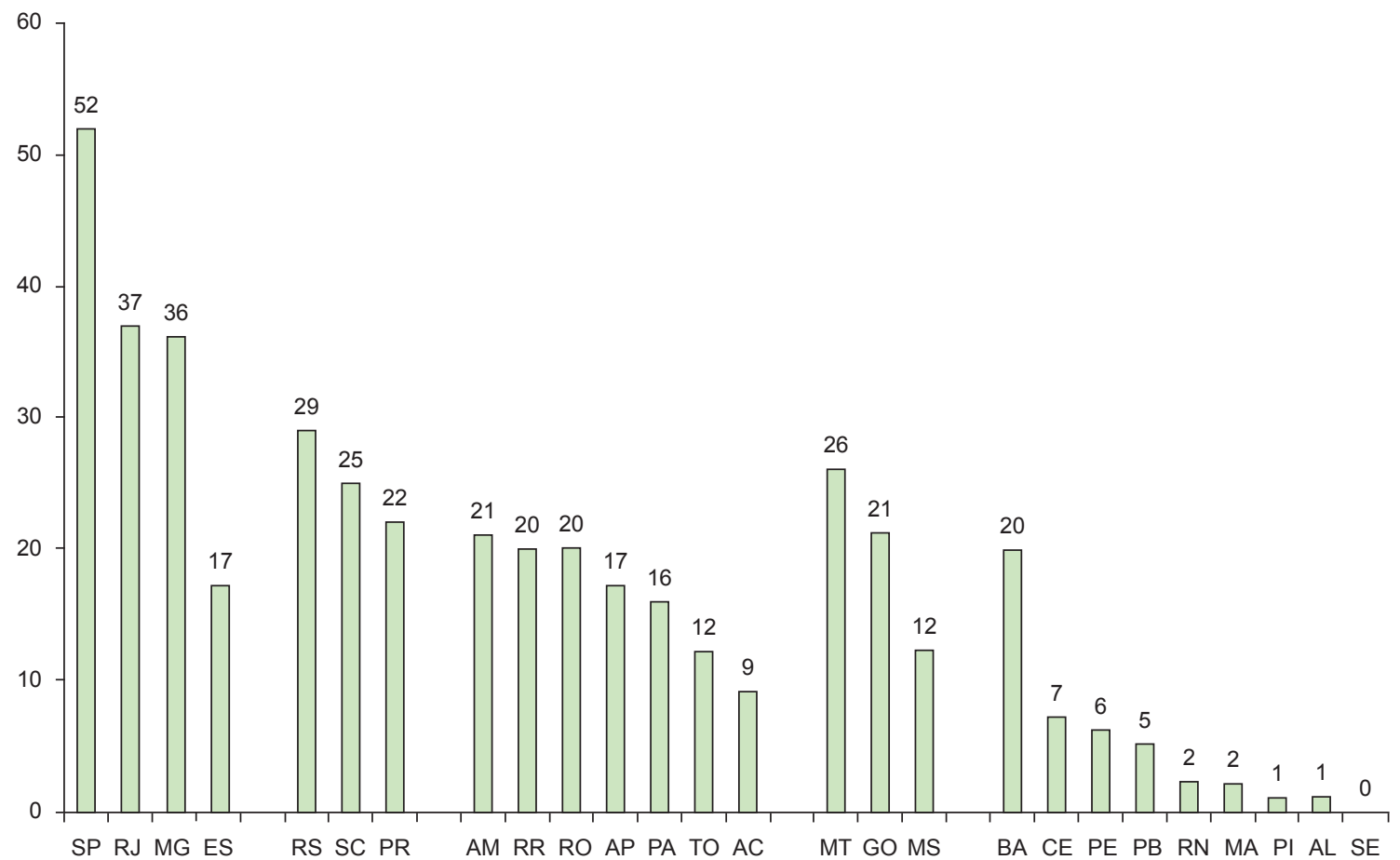

Figura 1. Número de espécies de Simuliidae registradas nos estados do Brasil.

Figure 1. Number of Simuliidae species recorded for each State of Brazil.

\section{Principais avanços relacionados ao Programa BIOTA/FAPESP}

Durante o Programa BIOTA/FAPESP, houve a descoberta e descrição de 2 novas espécies de Simuliidae, uma coletada no Pico dos Marins (Simulium marins Pepinelli, Hamada \& Currie, 2009) e outra coletada em Joanópolis (Simulium duodenicornium Pepinelli, Hamada \& Trivinho-Strixino, 2005). Outras 10 espécies foram coletadas pela primeira vez, aumentando em $30 \%$ o número de espécies de borrachudos registradas no estado de São Paulo.

\section{Os principais grupos de pesquisa de Simuliidae no Brasil são:}

- Grupo de pesquisa da Dra. Neusa Hamada do Instituto Nacional de Pesquisas da Amazônia (INPA);
- Grupo de pesquisa da Dra. Marilza Maia-Herzog da FIOCRUZ do Rio de Janeiro;

- Grupo de pesquisa do Dr. Mateus Pepinelli, pós-doutorando do Laboratório de Entomologia Aquática da USP Ribeirão Preto;

- Grupo de pesquida do Dr. Victor Py-Daniel do Instituto Nacional de Pesquisas da Amazônia (INPA).

3. As principais coleções de Simuliidae do Brasil são:

- Coleção do Instituto Nacional de Pesquisas da Amazônia (INPA);

- Coleção do Museu de Zoologia da Universidade de São Paulo (USP);

- Coleção da Faculdade de Saúde Pública da Universidade de São Paulo (FSP / USP); 
- Coleção de Simulídeos do Instituto Oswaldo Cruz, Fundação Oswaldo Cruz, Rio de Janeiro, Brazil (CSIOC antiga CLSO/IOC);

- Coleção do Museu de História Natural de Londres (Department of Entomology, Natural History Museum, London, United Kingdom - BMNH).

\section{Principais lacunas do conhecimento}

As principais lacunas do conhecimento estão relacionadas à biologia, comportamento, alimentação, capacidade vetorial de doenças em animais, potencial biotecnológico, biomonitoramento e os mecanismos de especiação que formam os complexos de espécies de Simuliidae.

\section{Perspectivas de pesquisa para os próximos 10 anos}

Integração de estudos moleculares e citotaxonômicos para desvendar e compreender os complexos de espécies. Construção da biblioteca de DNA Barcodes para as espécies de Simuliidae da região Neotropical e, consequentemente, do Estado de São Paulo e Brasil. Esse projeto já está em andamento e está sendo fomentado pela Fapesp no âmbito do projeto BIOTA. Com o auxílio de DNA barcoding, foi descrita recentemente uma nova espécie de Simuliidae para o Estado de Goiás (Hamada et al. 2010).

\section{Referências Bibliográficas}

ADLER A.H., CURRIE, D.C. \& WOOD, D.M. 2004. The Black Flies (Simuliidae) of North America. Connel University Press, Ithaca, New York, 941p.

ADLER, P. \& CROSSKEY, R.W. 2010. World Black Flies (Diptera: Simuliidae): a comprehensive revision of the taxonomic and geographical inventory. http://entweb.clemson.edu/biomia/pdfs/blackflyinventory.pdf (último acesso em 10/10/2010). 112p.
COSCARÓN, S. \& COSCARÓN-ARIAS, C.L. 2007. Neotropical Simuliidae (Diptera: Simuliidae). In Aquatic Biodiversity in Latin America (ABLA) (J. Adis, J.R. Arias, G. Rueda-Delgado \& K.M. Wantzen (Eds.). Pensoft, Sofia-Moscow, v.3, 685p.

CURRIE, D.C. \& ADLER, P.H. 2008. Global diversity of back flies (Diptera: Simulidiae) in freshwater. Hydrobiologia 595:469-475. http://dx.doi. org/10.1007/s10750-007-9114-1

HAMADA, N., McCREADIE, J.W. \& ADLER, P.H. 2002. Species richness and spatial distribution of blackflies (Diptera: Simuliidae) in streams of Central Amazonia, Brazil. Fresh Biol 47:31-40. http://dx.doi.org/10.1046/ j.1365-2427.2002.00778.x

HAMADA, N., PEPINELLI, M., MATTOS, A.M. \& LUZ, S.L.B. 2010. A new black fly species from Brazil, closely related to Simulium guianense Wise (Diptera, Simuliidae), revealed by morphology and DNA barcoding. Zootaxa 2428:22-36.

MALMQVIST, B., WOTTON, R.S. \& ZHANG, Y. 2001. Suspension feeders transform massive amounts of seston in large northern rivers. Oikos 92:35-43. http://dx.doi.org/10.1034/j.1600-0706.2001.920105.x

MALMQVIST, B., ADLER, P.H., KUUSELA, K., MERRITT, R.W. \& WOOTON, R.S. 2004. Black flies in the boreal biome, key organisms in both terrestrial and aquatic environments: a review. Écoscience 11:187-200.

STRIEDER, M.N. 2002. Diversidade e distribuição de Simuliidae (Diptera, Nematocera) no gradiente longitudinal da bacia do rio Maquiné, RS, Brasil. Biociências 10:127-137.

STRIEDER, M.N., SANTOS, J.E. \& PES, A.M.O. 2002. Diversidade e distribuição de Simuliidae (Diptera, Nematocera) no gradiente longitudinal da bacia do rio dos Sinos, no Rio Grande do Sul, Brasil. Entomol. Vectores 9:527-540. 
Pepinelli, M.

\section{Apêndice}

Apêndice 1. Referências complementares.

Appendix 1. Complementary bibliographic references.

ALENCAR, Y.B., LUDWIG, T.A.V., SOARES, C.C. \& HAMADA, N. 2001. Stomach content analyses of Simulium perflavum Roubaud 1906 (Diptera: Simuliidae) larvae from streams in Central Amazônia, Brazil. Mem Inst Oswaldo Cruz 96:561-576. http://dx.doi.org/10.1590/S0074-02762001000400020

CHARALAMBOUS, M., SHELLET, A.J. \& ARZUBE, M. 1993. The distribution and taxonomic status of chromosomal forms of the onchocerciasis vector Simulium exiguum (Diptera: Simuliidae). Med Vet Entomol 7:299-305. PMid:8369569. http://dx.doi.org/10.1111/j.1365-2915.1993.tb00694.x

CHARALAMBOUS, M., SHELLET, A.J., MAIA-HERZOG, M. \& LUNA DIAS, A.P.A. 1996. Four new cytotipes of the onchocerciasis vector blackfly Simulium guianense in Brasil. Med Vet Entomol 10:111-120. PMid:8744702. http://dx.doi.org/10.1111/j.1365-2915.1996.tb00716.x

COSCARÓN, S. 1980. Notas sobre Simúlidos Neotropicales IX. Sobre un grupo de especies próximo ao subgénero Simulium (Inaequalium) (Diptera-Insecta). Rev Soc Entomol Argentina 39(3):293-302.

COSCARÓN, S. 1982. Notas sobre simulidos Neotropicales X. Sobre un grupo peculiar de especies del genero Simulium del S.E. de Brasil, Paraguay y N.O. de Argentina (Diptera-Insecta). Rev Soc Entomol Argentina 41:65-76.

COSCARÓN, S. 1984. Revisión del Subgénero Simulium (Ectmnaspis) Enderlein (Simuliidae, Diptera, Insecta). Rev Soc Entomol Argentina 43(1):238-325.

COSCARÓN, S. 1987. El género Simulium Latreille en la región Neotropical: análisis de los grupos supraespecíficos, especies que los integran y distribución geográfica (Simuliidae, Diptera). Museu Paraense Emilio Goeldi, Coleção Emilie Snethlage, Belém, Pará, 111p.

COSCARÓN, S. 1990. Taxonomia y distribución del subgénero Simulium (Ectmnaspis) Enderlein (Simuliidae, Diptera, Insecta). Iheringia 70:109-170.

COSCARÓN, S. 1991. Fauna de água dulce de la República Argentina. Insecta, Diptera, Simuliidae 38. FECIC, Buenos. Aires, 304p.

COSCARÓN, S. \& COSCARÓN-ARIAS, C,L, 1997. Cladistic analysis of the subgenera Inaequalium, Psaroniocompsa and Chirostilbia of the genus Simulium, with comments on their distribution (Diptera: Simuliidae). Rev Soc Entomol Argentina 56:109-121.

COSCARÓN, S. \& WYGODZINSKY, P. 1984. Notas sobre Simúlidos Neotropicales VII. Sobre los subgéneros Psaroniocompsa Enderlein y Inaequalium, subgen. nov. Arq Zool 31:37-103.

CROSSKEY, R.W. \& HOWARD, T.M. 1997. A new taxonomic and geographical inventory of world blackflies (Diptera: Simuliidae). The Natural History Museum, London, 144p.

CROSSKEY, R.W. 1990. The Natural History of Blackflies. John Wiley \& Sons; The British Museum of Natural History, London, 711p.

CROSSKEY, R.W. \& HOWARD, T.M. 2002. Second update to the taxonomic and geographical inventory of world blackflies (Diptera: Simuliidae). The Natural History Museum, London, 14p.

CROSSKEY, R.W. \& HOWARD, T.M. 2004. A revised taxonomic and geographical inventory of world blackflies (Diptera: Simuliidae). The Natural History Museum, London. Available from http://www.nhm.ac.uk/entomology/projects/blackflies/Inventory.pdf (último acesso em 25/07/2006).

D’ANDRETTA, C. 1954. Sobre Simulium distinctum Lutz (1910) (Diptera, Simuliidae). Anais da Faculdade de Farmácia e Odontologia da Universidade de São Paulo 12:63-66.

D’ANDRETTA, C. 1956. Redescrição de Simulium perflavum Roubaud, 1908. Anais da Faculdade de Farmácia e Odontologia da Universidade de São Paulo 13:33-38.

D'ANDRETTA, M.A.V. \& D'ANDRETTA JUNIOR, C. 1946. As espécies neotropicais da família Simuliidae Schiner, 1864 (Diptera-Nematocera). I. Simulium (Eusimulium) orbitale Lutz, 1910. Simulium (Eusimulium) pintoi n.sp. e Simulium nigrimanum Macquart, 1837, sp. inquirendae. Mem Inst Oswaldo Cruz 43(1):85-152. http://dx.doi.org/10.1590/S0074-02761945000400004

D'ANDRETTA, M.A.V. \& D'ANDRETTA JUNIOR, R.C. 1947 Espécies neotropicais da família Simuliidae Schiner (Diptera Nematocera). Papéis Avulsos Dep Zoo 8(13): 145-180.

D'ANDRETTA, M.A.V. \& D’ANDRETTA JUNIOR, C. 1948. Espécies neotropicais da família Simuliidae Schiner (Diptera Nematocera). IV. Lutzsimulium cruzi Andr. \& Andr., 1946: descrição do alótipo macho e da larva. Papéis Avulsos Dep Zoo 5(10):637-648.

D’ANDRETTA, M.A.V. \& D’ANDRETTA JUNIOR, C. 1949. Espécies Neotropicais da Familia “Simuliidae” Schiner (Diptera, Nematocera). V. Redescrição do "Simulium auristriatum" Lutz, 1910 e "Simulium (Eusimulium) venustum infuscata" Lutz, 1909 var. inquirendae. Rev Bras Biol 9(1):55-66. PMid:18129507.

D’ANDRETTA, M.A.V. \& D’ANDRETTA JUNIOR, C. 1950. Espécies Neotropicais da família Simuliidae Schiner (Diptera, Nematocera). IV. Redescrição de Simulium pertinax Kollar, 1832. Papéis Avulsos Dep Zoo 9:193-213.

D’ANDRETTA, M.A.V. \& D’ANDRETTA JUNIOR, C. 1952. Espécies Neotropicais da família Simuliidae (Diptera, Nematocera). VII. Redescrição de Lutzsimulium pernigrum (Lutz, 1910), n. comb. e considerações sobre o gênero. Papéis Avulsos Dep Zoo 10:307-324.

D’ANDRETTA, M.A.V. \& DOLORES GONZÁLEZ, B.D. 1962. S. (Thyrsopelma) itaunense. Iheringia 65:95-102.

D’ANDRETTA, C. \& GONZÁLES, B.D. 1964. Três novas espécies brasileiras de Simuliidae (Diptera Nematocera). Mem Inst Butantan 30:103-116.

GERAIS, B.B. \& RIBEIRO, T.C. 1986. Relatos de casos Oncocercose: primeiro caso autóctone da região centro-oeste do Brasil. Rev Soc Bras Med Tropical 19(2):105-107. PMid:3432620.

GIL-AZEVEDO, L.R., SANTOS MALLET, J.R. \& MAIA-HERZOG, M. 2004. Caracteres diagnósticos de Simulium (Chirostilbia) pertinax Kollar (Diptera: Simuliidae). Neot Entomol 33(4):433-437. http://dx.doi.org/10.1590/S1519-566X2004000400006

GIL-AZEVEDO, L.R., FIGUERÓ, R. \& MAIA-HERZOG, M. 2005. Simulium (Psaroniocompsa) stellatum (Diptera: Simuliidae), a new black fly from a high mountain range in southeastern Brazil. Zootaxa 922:1-12.

HAMADA, N. 1997. Cytotaxonomy and ecology of four species in the Simulium perflavum species group and associated black flies in Central Amazonia, Brazil. PhD Thesis, Clemson University, Clemson, South Carolina.

HAMADA, N. 1998. Bionomics on Simulium perflavum Roubaud (Diptera: Simuliidae) in Central Amazonia, Brazil. Rev Bras Entomol 41(2):523-526.

HAMADA, N. \& ADLER, P.H. 1998. Taxonomy of the Simulium perflavum species-group (Diptera: Simuliidae) with description of a new species from Brazil. Insecta Mundi 12:207-226. 
Checklist de Simuliidae de São Paulo

HAMADA, N. \& ADLER, P.H. 1999. Cytotaxonomy of four species in the Simulium perflavum species-group (Diptera: Simuliidae) from Brazilian Amazonia. Syst Entomol 24:273-288. http://dx.doi.org/10.1046/j.1365-3113.1999.00080.x

HAMADA, N. \& PEPINELLI, M. 2004. Simulium (Chirostilbia) bifenestratum (Diptera, Simuliidae), a new black-fly species from the Atlantic Forest, São Paulo State, Brazil. Mem Inst Oswaldo Cruz 99:45-52. http://dx.doi.org/10.1590/S0074-02762004000100008

HAMADA, N., ALE-ROCHA, R. \& LUZ, S.B.L. 2003. Description of Simulium damascenoi (Diptera: Simuliidae) male and the black-fly species from the State of Amapá, Brazil. Mem Inst Oswaldo Cruz 98:353-360. http://dx.doi.org/10.1590/S0074-02762003000300010

HAMADA, N., HERNANDEZ, L.M., LUZ, S.L.B. \& PEPINELLI, M. 2005. Simulium (Chirostilbia) jefersoni, new species of black fly (Diptera: Simuliidae) from the State of Bahia, Brazil. Zootaxa 1123:21-37.

HAMADA, N., PEPINELLI, M. \& MARDINI, L.B.L.F. 2006. Rio Grande do Sul. Secretaria Estadual da Saúde. Centro Estadual de Vigilância em Saúde. Simulídeos: Programa Estadual do Rio Grande do Sul, Brasil: chave de identificação de pupas da família Simuliidae (Diptera, Nematocera) para apoio às equipes regionais e municipais na determinação das espécies. CEVS, Porto Alegre, 40p.

HERNÁNDEZ, L.M. \& SHELLEY, A.J. 2005. New specific synonymies and taxonomic notes on Neotropical blackflies (Diptera: Simuliidae). Zootaxa 853:1-46.

HERNÁNDEZ, L.M. \& SHELLEY, A.J., LUNA DIAS, A.P.A. \& MAIA-HERZOG, M. 2005. Redescription of the female, male and pupa of Simulium itaunense D'Andretta \& González B. (Diptera: Simuliidae). Zootaxa 896:1-14.

HERNÁNDEZ, L.M., SHELLEY, A.J., LUNA-DIAS, A.P.A. \& MAIA-HERZOG, M. 2007a. New specific synonymies and taxonomic notes on Neotropical black flies (Diptera: Simuliidae) belonging to the subgenera Chirostilbia Enderlein, Hemicnetha Enderlein, Inaequalium Coscarón \& Wygodzinsky, Psaroniocompsa Enderlein and Psilopelmia Enderlein. Zootaxa 1506:1-80.

HERNÁNDEZ, L.M., SHELLEY, A.J., LUNA-DIAS, A.P.A. \& MAIA-HERZOG, M. 2007b. Review of the Neotropical blackfly subgenus Inaequalium Coscarón \& Wygodzinsky (Diptera: Simuliidae) based on adults and pupal morphology. Zootaxa 1649:1-96.

LANDEIRO, V.L., PEPINELLI, M. \& HAMADA, N. 2009. Species Richness and Distribution of Blackflies (Diptera: Simuliidae) in the Chapada Diamantina Region, Bahia, Brazil. Neot Entomol 38(3):332-339. PMid:19618048. http://dx.doi.org/10.1590/S1519-566X2009000300006

LANE, J. \& PORTO, C.E. 1939. Simulideos da região neotropical: o gênero Eusimulium. Bol Biológico 4(2):168-176.

LANE, J. \& PORTO, C.E. 1940. Simulídeos da região Neotrópica. III. Descrição de novas espécies dos gêneros "Simulium "Eusimulium”. Arq Inst Biológico 11:189-195.

LUTZ, A. 1909. Contribuição para o conhecimento das espécies Brasileiras do gênero "Simulium". Memórias do Instituto Oswaldo Cruz 1:124-146. http:// dx.doi.org/10.1590/S0074-02761909000200006

LUTZ, A. 1910. Segunda contribuição para o conhecimento das espécies Brasileiras do gênero "Simulium”. Mem Inst Oswaldo Cruz 2:213-267.

LUTZ, A. 1922. Zoologia medica. Nematóceros hematófagos não pertencente aos culicídeos. A Folha Médica 3:89-92.

MAIA-HERZOG, M., SHELLEY, A.J., LUNA DIAS, A.P.A. \& MALAGUTI, R. 1984. Comparação entre Simulium brachycladum e Simulium rubrithorax, suas posições no sungênero Hemicnetha e nota sobre uma espécie próxima S. scutristriatum (Diptera: Simuliidae). Mem Inst Oswaldo Cruz 79:342-356.

MAIA-HERZOG, M., SHELLEY, A.J. \& LUNA DIAS, A.P.A. 1985. Simulium hirtipupa Lutz, 1910 (Diptera: Simuliidae). Descrição dos adultos e larva e redescrição da pupa. Mem Inst Oswaldo Cruz 80(4):483-490. http://dx.doi.org/10.1590/S0074-02761985000400015

MATTOS, A.A. 2007. Comparação molecular e citológica de diferentes populações geográficas de Simulium guianense Wise s.l., no Brasil. Dissertação de mestrado, Universidade Federal do Amazonas, Manaus, 96p.

MORAIS, M.A.P. \& CHAVES, G.M. 1974. Oncocercose no Brasil. Novos achados entre os índios Ianomâmis. Bol Ofic Sanit Panamá 1-5.

MOREIRA, G.R.P., PEGORARO, R.A. \& SATO, G. 1994. Influência de fatores abióticos sobre o desenvolvimento de Simulium nogueirai D’Andretta \& González em um córrego de Mata Atlântica. Anais da Soc Entomol Brasil 23(3):525-542.

PEPINELLI, M., STRIXINO, S.T. \& HAMADA, N. 2003. Description of Simulium (Chirostilbia) friedlanderi Py-Daniel, 1987 (Insecta: Diptera: Simuliidae) female. Biota Neotrop.: http://www.biotaneotropica.org.br/v3n2/pt/abstract?short-communication+ BN00203022003 (último acesso em 12/02/2005).

PEPINELLI, M., HAMADA, N. \& TRIVINHO-STRIXINO, S. 2005. Simulium (Thyrsopelma) duodenicornium, a new black fly species (Diptera: Simuliidae) from the Southeast Region of Brazil. Zootaxa 1040:17-29.

PEPINELLI, M., HAMADA, N. \& TRIVINHO-STRIXINO, S. 2006. Larval description of Simulium (Notolepria) cuasiexiguum and Simulium (Chirostilbia) obesum and new records of black fly species (Diptera: Simuliidae) in the states of São Paulo and Minas Gerais, Brazil. Neot Entomol 35:698-704. PMid:17144144. http://dx.doi.org/10.1590/S1519-566X2006000500019

PEPINELLI, M. (2008) Simuliidae (Diptera, Nematocera) do Estado de São Paulo. Mateus Pepinelli, tese de doutorado - São Carlos: UFSCar, 144 f.

PESSOA, F.A.C., RIOS-VELÁSQUEZ, C.M. \& PY-DANIEL, V. 2005. First survey of Simuliidae (Diptera) from the North of Ceara State, Brazil, with description of a new species and identification keys for the immature stages. Zootaxa 1051:1-18.

PY-DANIEL, V. \& BARBOSA, U.C. 2007. Simuliidae (Diptera, Culicomorpha) no Brasil - XIII sobre o Inaequalium lundi sp. n. Acta Amaz 37(3):465-474. http://dx.doi.org/10.1590/S0044-59672007000300019

PY-DANIEL, V. \& COSCARÓN, S. 1982. Simuliidae (Diptera: Nematocera) no Brasil. I. Sobre o Simulium rappae sp. n. Rev Brasil Biol 42(1):155-163.

PY-DANIEL, V. \& MOREIRA, G.R.P. 1988. Simuliidae (Diptera, Culicomorpha) no Brasil VII. Sobre o Simulium (Chirostilbia) empascae sp. n. Iheringia 67:77-86.

PY-DANIEL, V. \& MOREIRA, G.R.P. 1989. Simuliidae no Brasil (Diptera, Culicomorpha). IX. Simulium (Inaequalium) nogueirai D’Andretta \& González, 1964. Iheringia 69:85-95.

PY-DANIEL, V. \& SAMPAIO, R.T.M. 1994. Jalacingomyia gen. n. (Culicomorpha); a ressurreição de Gymnopaidinae; a eliminação do nível tribal; apresentação de novos caracteres e a redescrição dos estágios larval e pupal de Simulium columbachensis (Fabricius, 1787) (Diptera: Simuliidae). Memorias del CAÍCET 4:101-148.

PY-DANIEL, V. \& SHELLEY, A.J. 1980. Revisão do Simulium spinibranchium Lutz, 1910 (Diptera: Simuliidae), com a primeira descrição dos adultos e larva, e redescrição da pupa. Acta Amaz 10(1):213-223.

PY-DANIEL, V. 1983. Caracterização de dois novos subgêneros em Simuliidae (Diptera, Culicomorpha) Neotropicais. Amazoniana 8(2):159-223. 
Pepinelli, M.

PY-DANIEL, V. 1987. Simuliidae (Diptera, Culicomorpha) no Brasil. V. Sobre o Simulium (Chirostilbia) friedlanderi sp. n. e revisão de Simulium (C.) laneportoi Vargas, 1941. Rev Saúde Pública 21(4):331-341. http://dx.doi.org/10.1590/S0034-89101987000400007

PY-DANIEL, V. 1989. Novas sinonimias e correções em Simulium com a revalidação de S. pruinosum Lutz, 1904 (Culicomorpha, Simuliidae). Revista de Saúde Pública 23(3):254-257. http://dx.doi.org/10.1590/S0034-89101989000300011

PY-DANIEL, V., KONRAD, H.G. \& GASTAL, H.A.O. 1985. Simuliidae (Diptera, Culicomorpha) no Brasil. IV. Descrição das larvas de Simulium (Psaroniocompsa) brevifurcatum Lutz, 1910; S. (P.) anamariae Vulcano, 1962; S. (Thyrsopelma) itaunense D’Andretta \& Dolores Gonzales, 1962. Iheringia 65:95-102.

PY-DANIEL, V., SOUZA, M.A.T. \& CALDAS, E.P. 1988. Simuliidae (Diptera, Culicomorpha) no Brasil. III. Sobre o Simulium (Chirostilbia) riograndense sp. n. e revisão do Simulium (Chirostilbia) distinctum Lutz, 1910. Inheringia 67:37-57.

RIOS-VELÁSQUEZ, C., HAMADA, N. \& ADLER, P.H. 2002. Cytotaxonomy of Simulium goeldi Cerqueira \& Nunes de Mello and Simulium ulyssesi in Central Amazonia, Brazil. Insect Syst Evol 33:113-120. http://dx.doi.org/10.1163/187631202X00082

SATO, G. 1987. Identificação de peixes predadores de larvas de simulídeos da região de Joinville/SC. Ciência e Cultura 39(10):962-966.

SHELLEY, A.J. \& COSCARÓN, S. 2001. Simuliid Blackflies (Diptera: Simuliidae) and Ceratopogonid Midges (Diptera: Ceratopogonidae) as vectors of Mansonella ozzardi (Nematoda: Onchocercidae) in Northern Argentina. Mem Inst Oswaldo Cruz 96(4):451-458. http://dx.doi.org/10.1590/S007402762001000400003

SHELLEY, A.J., PINGER, R.R., MORAES, M.A.P., CHARLWOOD, J.D. \& HAYES, J. 1979. Vectors of Onchocerca volvulus at the river Toototobi, Brazil. Journal of Helminthology 53:41-43. http://dx.doi.org/10.1017/S0022149X00005721

SHELLEY, A.J., PINGER, R.R. \& MORAES, M.A.P. 1982. The taxonomy, biology and medical importance of Simulium amazonicum Goeldi (Diptera: Simuliidae), with a review of related species. Bull British Museum (Natural History) 44(1):1-29.

SHELLEY, A.J., LUNA DIAS, A.P.A. \& MAIA-HERZOG, M. 1984. New specific synonimy in Neotropical Simulium s.1. (Diptera: Simuliidae). Memórias do Instituto Oswaldo Cruz 79(2):143-161. http://dx.doi.org/10.1590/S0074-02761984000200001

SHELLEY, A.J., LOWRY, C.A., MAIA-HERZOG, M., LUNA-DIAS, A.P.A. \& MORAES, M.A.P. 1997. Biosystematic studies on the Simuliidae (Diptera) of the Amazonia onchocerciasis focus. Bull Nat History Museum 66:1-120.

SHELLEY, A.J., MAIA-HERZOG, M., LOWRY, C.A., LUNA DIAS, A.P.A., GARRITANO, P.R., SHELLEY, A., CAMARGO, M. \& CARTER, H.G. 2000. The Simuliidae (Diptera) of the secondary onchocerciasis focus at Minaçu in central Brazil. Bull Nat History Museum (Entomology series) 69:171-221.

SHELLEY, A.J., LUNA DIAS, A.P.A., MAIA-HERZOG, M., LOWRY, C.A., GARRITANO, P.R., PENN, M. \& CAMARGO, M. 2001. Simulium cuasiexiguum, a new blackfly species (Diptera: Simuliidae) from the Minaçu Area in the State of Goiás, Central Brazil. Memórias do Instituto Oswaldo Cruz 96:483-496. http://dx.doi.org/10.1590/S0074-02762001000400008

STRIEDER, M.N. 1998. Revisão e análise cladística de espécies de Inaequalium (Coscarón \& Wygodzinsky, 1984) (Diptera: Simuliidae). Phd Thesis, Pontifícia Universidade Católica de Rio Grande do Sul, Porto Alegre.

STRIEDER, M.N. \& PY-DANIEL, V. 1999. Espécies de Inaequalium (Diptera, Simuliidae), dados bionômicos e chaves para sua identificação. Biociências $7(2): 43-72$

STRIEDER, M.N. \& PY-DANIEL, V. 2000. Revisão de Inaequalium (Diptera, Simuliidae), com redescrição das formas imaturas e descrição de uma nova espécie. Entomol Vect 7(2):1-91.

STRIEDER, M.N. \& PY-DANIEL, V. 2002. A cladistic Analysis of Inaequalium (Coscarón \& Wygodzinsky, 1984), with Information on Geographical Distribution (Diptera: Simuliidae). Mem Inst Oswaldo Cruz 97(1):65-72. http://dx.doi.org/10.1590/S0074-02762002000100010

VULCANO, M.A. 1959. Descrição de Simulium obesum, sp. n. (Diptera, Simuliidae). Pap Avulsos do Depart Zool 13:351-360.

VULCANO, M.A. 1962. Simulídeos da Serra da Bocaína. Observações bionômicas, revalidação de Simulium brevifurcatum Lutz e descrição de uma espécie nova, S. anamariae (Diptera, Simuliidae). Pap Avulsos do Depart Zool 15:239-272.

WYGODZINSKY, P. 1950. Contribución al conocimento de los Simulidos Argentinos. III. Simulium dinellii (Joan, 1912) y S. wolffhuegeli (Enderlein, 1920). Anal Inst Medicina Regional 3(1):75-97.

WYGODZINSKY, P. 1951. Sobre Simulium jujuyense Paterson \& Shannon, 1927, Simulium exiguum Roubaud, 1906 y Simulium opalinifrons (Enderlein, 1934). Anal Inst Medicina Regional 3(2):207-220. 